\title{
Clinical Characteristics of Patients with Severe and Critical COVID-19 in Wuhan: A Single-Center, Retrospective Study
}

\author{
Zhaohui Chen · Junyi Hu • Lilong Liu · Youpeng Zhang • \\ Dandan Liu $\cdot$ Ming Xiong $\cdot$ Yi Zhao $\cdot$ Ke Chen $\cdot$ Yu-Mei Wang
}

Received: September 4, 2020 / Accepted: November 26, 2020 / Published online: January 22, 2021

(C) The Author(s) 2021

\begin{abstract}
Introduction: This retrospective, single-center study was performed to systemically describe the characteristics and outcomes of patients with severe and critical coronavirus disease 2019 (COVID-19) in Wuhan, analyze the risk factors, and propose suggestions for clinical diagnosis and treatment to guide the subsequent clinical practice.

Methods: A total of 753 consecutive patients with COVID-19 admitted to the West Campus of Wuhan Union Hospital from January 22, 2020 to May 7, 2020 were enrolled in this study.
\end{abstract}

Zhaohui Chen, Junyi Hu, Lilong Liu, Youpeng Zhang, and Dandan Liu contributed equally to this work.

Supplementary Information The online version contains supplementary material available at https:// doi.org/10.1007/s40121-020-00379-2.

Z. Chen · J. Hu $\cdot$ L. Liu $\cdot$ Y. Zhang $\cdot$ D. Liu

M. Xiong $\cdot$ K. Chen $(\bowtie)$

Department of Urology, Union Hospital, Tongji

Medical College, Huazhong University of Science

and Technology, Wuhan 430022, China

e-mail: shenke@hust.edu.cn;

wangyumei75@163.com

Y. Zhao · Y.-M. Wang (凶)

Department of Nephrology, Union Hospital, Tongji

Medical College, Huazhong University of Science

and Technology, Wuhan 430022, China

e-mail: shenke@hust.edu.cn;

wangyumei75@163.com
Demographic, clinical, laboratory, and outcome data were extracted from the electronic medical records of Wuhan Union Hospital and were exhaustively analyzed using R (version 3.6.1).

Results: A total of 493 severe and 228 critical cases out of 753 COVID-19 cases were considered in this study. Among the critical cases, the death rate was $79.4 \%$, and age was a risk factor for death. Compared to the severe disease group, the critical disease group had higher white blood cell (WBC) and neutrophil counts and a decreased lymphocyte count at admission. Compared to early death cases (death within 1 week after admission), a more prolonged course of the disease was associated with a higher risk of hypoproteinemia, liver injury, thrombocytopenia, anemia, disseminated intravascular coagulation (DIC), coagulation disorders, acute kidney injury (AKI), and infection. Higher creatine kinase (CK) and lactate dehydrogenase (LDH) levels were related to early death events, but univariate and multivariate analyses confirmed only $\mathrm{LDH}$ as an independent predictor of early death. Notably, anticoagulation therapy was associated with an improved prognosis of critical cases in this cohort.

Conclusion: Our results showed large differences between patients with severe and critical COVID-19. During the course of COVID-19 in the critical disease group, the incidence of hypoproteinemia, anemia, thrombocytopenia, and coagulation disorders increased 
significantly, which highlighted the importance of medical care in the first week after admission. $\mathrm{LDH}$ could act as an independent predictor of early death in critical cases, and anticoagulation therapy was correlated with an improved prognosis of patients with critical COVID-19.

Keywords: Anticoagulation; COVID-19; Critical; Early death; Medical care

\section{Key Summary Points}

\section{Why carry out this study?}

Outbreak of the COVID-19 pandemic has evolved into one of the most serious public health events.

To systemically analyze clinical features and determine risk factors between patients with severe and critical COVID19.

\section{What was learned from the study?}

Large differences between patients with severe and critical COVID-19.

Anticoagulation therapy was correlated with improved prognosis of patients with critical COVID-19.

LDH is an independent predictor of early death in critical cases.

\section{DIGITAL FEATURES}

This article is published with digital features, including a summary slide to facilitate understanding of the article. To view digital features for this article go to https://doi.org/10.6084/ m9.figshare.13286585.

\section{INTRODUCTION}

Since December 2019, coronavirus disease 2019 (COVID-19) caused by severe acute respiratory syndrome coronavirus 2 (SARS-CoV-2) has spread to become a global pandemic. As of October 10, 2020, there were over 36 million confirmed cases and 1,056,186 deaths [1], with the number still surging worldwide. With the continuous increase in COVID-19 cases, social distancing and screening for infected persons are still necessary [2]. Protection of high-risk populations is still a challenge.

According to their clinical manifestations, patients with COVID-19 can be divided into severe and non-severe groups. The severe group could be further divided into severe and critical subgroups. In the published case series, patients with non-severe disease had a favorable prognosis. However, the mortality of severe cases, especially critical cases, is still high [3]. Estimation of monitoring indicators and therapeutic targets for severe cases could help physicians choose appropriate treatment strategies.

Several retrospective cohort studies from Wuhan have reported that older age, male sex, and comorbidities are risk factors for COVID-19 [3-5]. Cohorts from other countries have also been reported [6-8]. Most of these studies estimating risk factors grouped all of the survivors together, but there is a large difference between survivors with mild disease and critical diseases.

To systemically estimate more intuitive risk factors and to search for monitoring indicators of critical cases to propose a diagnosis and treatment recommendation for patients with COVID-19 to guide subsequent clinical practice, we systemically describe the characteristics and outcomes of 753 patients hospitalized at West Campo of Wuhan Union Hospital from January 22, 2020 to May 7, 2020. This hospital was used as an intensive care center at the peak of the pandemic in Wuhan. These findings could help physicians recognize high-risk patients among severely and critically ill patients with COVID-19 and then make early interventions.

\section{METHODS}

\section{Study Design and Participants}

The retrospective cohort study included all 753 participants hospitalized at West Campus of 
Wuhan Union Hospital from January 22, 2020 to May 7, 2020. All patients were diagnosed according to the Diagnosis and Treatment Protocol for Novel Coronavirus Pneumonia (Trial Version 7) published by the National Health Commission (www.gov.cn/zhengce/zhengceku/ 2020-03/04/content_5486705.htm). In this cohort, only 32 patients had non-severe disease throughout the course of the disease. Definitive outcomes of all of these patients were observed.

The suspected cases had one of the following pieces of etiological or serological evidence and were diagnosed with COVID-19: (a) A positive result on an RT-PCR assay for SARS-CoV-2; (b) Viral gene sequencing shows high homology with SARS-CoV-2; (c) SARS-CoV-2-specific IgM and IgG antibodies were positive in the serum; (d) The serum SARS-CoV-2-specific IgG antibody changed from negative to positive or was elevated by at least fourfold during the recovery period compared with the acute phase.

Severe COVID-19 cases were defined as meeting any of the following criteria. For adults: (a) Shortness of breath, respiratory rate (RR) $\geq 30$ times/min; (b) Oxygen saturation $\leq 93 \%$ in the resting state; (c) Arterial partial pressure of oxygen $(\mathrm{PaO} 2) /$ fraction of inspired oxygen $(\mathrm{FiO} 2) \leq 300 \mathrm{mmHg} \quad(1 \mathrm{mmHg}=0.133 \mathrm{kPa})$; (d) Patients with pulmonary imaging showing significant lesion progression (>50\%) within 24-48 h. For children: (a) Shortness of breath ( $<2$ months old, $\quad \mathrm{RR} \geq 60$ times/min; 2-12 months old, $\mathrm{RR} \geq 50$ times/min; $1-5$ years old, $\mathrm{RR} \geq 40$ times/min; $>5$ years old, $\mathrm{RR} \geq 30$ times/min), excluding the influence of fever and crying; (b) Oxygen saturation $\leq 92 \%$ in the resting state; (c) Assisted breathing (moaning, nasal flapping, trident signs), cyanosis, intermittent apnea; (d) Lethargy and convulsions; (e) Refusal of food or difficulty in feeding, signs of dehydration.

Critical COVID-19 cases were defined as including at least one of the following conditions: (a) Respiratory failure requiring mechanical ventilation; (b) Shock; (c) Patients with other organ failure who needed to be monitored in the intensive care unit (ICU).

This retrospective study received approval from the Research Ethics Commission of Tongji Medical College, Huazhong University of
Science and Technology (S100). The study was performed in accordance with the Helsinki Declaration of 1964, and its later amendments.

\section{Data Collection}

Demographic, clinical, laboratory, and outcome data were extracted from electronic medical records of Wuhan Union Hospital. All data were checked by two physicians (Ming Xiong and Dandan Liu) and verified by a third physician (Yu-Mei Wang) to eliminate any potential mistakes.

\section{Statistical Analysis}

Continuous variables were presented as the median and interquartile range (IQR). The Mann-Whitney $U$ or Kruskal-Wallis tests were used to analyze continuous variables. Dunn's test was performed for pairwise comparisons and the $p$ value was adjusted by the Bonferroni method. Categorical variables were presented as frequencies and percentages. The $\chi^{2}$ test or Fisher's test was used to analyze categorical variables. Pairwise comparisons were made with the chisq.post.hoc function of the fifer package (FDR strategy). Univariate and multivariate logistic regression analyses were conducted to evaluate potential risk factors. The covariates included comorbidities, complications, treatment (invasive ventilation, extracorporeal membrane oxygenation (ECMO), continuous renal replacement therapy (CRRT), drugs, and corticosteroids), and biochemical indexes (routine blood tests, coagulation function, liver function, renal function, myocardial enzyme, and inflammation-related indicators). In this study, we mainly analyzed the biochemical indexes at the time of admission, 7 days after admission, 14 days after admission, and before the patients were discharged from the hospital or before the patients died. Serum ferritin was not analyzed because of too many missing values. A two-sided $p<0.05$ was considered as statistically significant. All analyses were conducted in $\mathrm{R}$ (version 3.6.1). 


\section{RESULTS}

This study involved 753 patients with confirmed COVID-19, including 32 moderate, 493 severe, and 228 critical cases. Here, we considered only severe and critical cases. For the severe and critical patients, the median age was 62 years old (IQR 51-69), ranging from 14 to 93 years (Table 1 ). Consistent with previous studies, older age was related to critical COVID19 cases $[9,10]$. A total of $54.2 \%$ of the participants were male, approximately half of the entire cohort. However, 155 out of 228 (68\%) critical cases were male, significantly higher than the proportion of female patients (32\%), indicating that male sex is a risk factor for critical disease, which was also reported by several previous studies $[4,11]$. Compared to severe cases, time from showing symptoms to admission was significantly shorter in the critical disease group. Patients with comorbidities were also more likely to become critical COVID-19 cases, and the critical cases tend to have more comorbidities when compared to the severe group (Table 1). In general, fever (75.2\%), cough $(69.6 \%)$, and fatigue $(52.7 \%)$ were the most prevalent symptoms, followed by dyspnea $(41.6 \%)$, myalgia (23\%), and diarrhea (17.5\%). Among these symptoms, fatigue and dyspnea were more prevalent in critical patients.

When investigating complications that occurred during hospitalization, we found that $61.5 \%$ of severe and $79.4 \%$ of critical cases had different levels of liver injury. Apart from that, all complications, including ARDS, heart injury, shock, and thrombocytopenia, were more prevalent in critical patients. Most of the severe cases (99.4\%) had oxygen inhalation during hospitalization, and none of them needed noninvasive or invasive ventilation. In contrast, $55.3 \%$ of the critical cases had non-invasive ventilation, and $45.2 \%$ required invasive ventilation.

When comparing biochemical indexes at admission, we found large differences between severe and critical cases. Except for hemoglobin, all other indexes collected in the analysis showed differences between severe and critical cases at the time of admission (Supplementary
Table 1). Compared to the severe group, the critical cases had higher WBC and neutrophil counts and decreased lymphocyte counts. For coagulation-related indicators, the critical disease group showed higher D-dimer, prolonged prothrombin time (PT), and activated partial thromboplastin time (APTT) and a decreased platelet count. Increases in aspartate transaminase (AST) and alanine transaminase (ALT) and decreases in total protein and albumin (ALB) were also observed, accounting for the higher liver injury incidence in critical cases. In addition, heart injury and kidney injury indicators, including lactate dehydrogenase (LDH), creatine kinase (CK), blood urea nitrogen (BUN), and serum creatinine (Scr), were all higher in the critical disease group. Higher C-reactive protein (CRP), procalcitonin (PCT), erythrocyte sedimentation rate (ESR), and serum ferritin level were also observed, indicating more severe inflammation in the critical disease group.

In this retrospective cohort, all 181 deaths emerged in the critical disease group, and the mortality rate was $79.4 \%$. To investigate what influenced the fate of these patients, we compared demographic, clinical, and laboratory information between survivors and non-survivors among the critical cases (Tables 2 and 3). We found that the non-survivors of critical cases were older than the survivors (68 vs. 63 years old). Notably, although male patients seem more likely to develop critical disease, once the disease progressed to a critical stage, the mortality risk was not significantly different between male and the female patients $(81.3 \%$ vs. $75.3 \%$ ). Comorbidities were also not associated with an increased death rate in this group. However, 20 critical cases with cancer all died, suggesting that cancer is a risk factor for death of patients with COVID-19. In addition, none of the prevalent symptoms were related to increased risk of death.

Regardless of the survivors or non-survivors of critical cases, acute respiratory distress syndrome (ARDS) occurred in almost all these cases. Shock, heart injury, thrombocytopenia, anemia, DIC, coagulation disorders, and AKI were significantly associated with unfavorable outcomes. Interestingly, a higher percentage of survivors suffered venous thrombosis, which 
Table 1 Demographic and clinical information of severe and critical cases

\begin{tabular}{|c|c|c|c|c|}
\hline & Total $(n=721)$ & Severe $(n=493)$ & Critical $(n=228)$ & $p$ value $^{*}$ \\
\hline Age & $62(51-69)$ & $58(48-66)$ & $67(60-76)$ & $<0.0001$ \\
\hline \multicolumn{5}{|l|}{ Sex } \\
\hline Male & $391(54.2 \%)$ & $236(47.9 \%)$ & $155(68 \%)$ & \\
\hline Female & $330(45.8 \%)$ & $257(52.1 \%)$ & $73(32 \%)$ & $<0.0001$ \\
\hline \multicolumn{5}{|l|}{ Course of disease } \\
\hline Time before admission & $11(7-15)$ & $12(8-15)$ & $10(7-15)$ & 0.0293 \\
\hline Hospitalization & $21(12-32)$ & $22(16-33)$ & $12(6-29)$ & $<0.0001$ \\
\hline Total & $35(24-47)$ & $37(28-48)$ & $26(17-42)$ & $<0.0001$ \\
\hline \multicolumn{5}{|l|}{ Comorbidities } \\
\hline Total $^{\mathrm{a}}$ & $459(63.7 \%)$ & $282(57.2 \%)$ & $177(77.6 \%)$ & $<0.0001$ \\
\hline Number & $1(0-2)$ & $1(0-2)$ & $1(1-2.5)$ & $<0.0001$ \\
\hline Diabetes & $136(18.9 \%)$ & $83(16.8 \%)$ & $53(23.2 \%)$ & 0.052 \\
\hline Hypertension & $245(34 \%)$ & $144(29.2 \%)$ & $101(44.3 \%)$ & 0.0001 \\
\hline Cancer & $47(6.5 \%)$ & $27(5.5 \%)$ & $20(8.8 \%)$ & 0.1324 \\
\hline Cardiac disease & $103(14.3 \%)$ & $56(11.4 \%)$ & $47(20.6 \%)$ & 0.0014 \\
\hline \multicolumn{5}{|l|}{ Symptoms } \\
\hline Fever & $542(75.2 \%)$ & $362(73.4 \%)$ & $180(78.9 \%)$ & 0.133 \\
\hline Cough & $502(69.6 \%)$ & $344(69.8 \%)$ & $158(69.3 \%)$ & 0.9658 \\
\hline Fatigue & $380(52.7 \%)$ & $247(50.1 \%)$ & $133(58.3 \%)$ & 0.0479 \\
\hline Dyspnea & $300(41.6 \%)$ & $175(35.5 \%)$ & $125(54.8 \%)$ & $<0.0001$ \\
\hline Myalgia & $166(23 \%)$ & $118(23.9 \%)$ & $48(21.1 \%)$ & 0.4474 \\
\hline Diarrhea & $126(17.5 \%)$ & $85(17.2 \%)$ & $41(18 \%)$ & 0.8901 \\
\hline \multicolumn{5}{|l|}{ Complications } \\
\hline Liver injury & $484(67.1 \%)$ & $303(61.5 \%)$ & $181(79.4 \%)$ & $<0.0001$ \\
\hline ARDS & $262(36.3 \%)$ & $36(7.3 \%)$ & $226(99.1 \%)$ & $<0.0001$ \\
\hline Heart injury & $183(25.4 \%)$ & $62(12.6 \%)$ & $121(53.1 \%)$ & $<0.0001$ \\
\hline Shock & $181(25.1 \%)$ & $0(0 \%)$ & $181(79.4 \%)$ & $<0.0001$ \\
\hline Thrombocytopenia & $135(18.7 \%)$ & $22(4.5 \%)$ & $113(49.6 \%)$ & $<0.0001$ \\
\hline AKI & $75(10.4 \%)$ & $7(1.4 \%)$ & $68(29.8 \%)$ & $<0.0001$ \\
\hline DIC & $75(10.4 \%)$ & $4(0.8 \%)$ & $71(31.1 \%)$ & $<0.0001$ \\
\hline \multicolumn{5}{|l|}{ Treatment } \\
\hline Oxygen inhalation & $668(92.6 \%)$ & $490(99.4 \%)$ & $178(78.1 \%)$ & $<0.0001$ \\
\hline Non-invasive ventilation & $126(17.5 \%)$ & $0(0 \%)$ & $126(55.3 \%)$ & $<0.0001$ \\
\hline
\end{tabular}


Table 1 continued

\begin{tabular}{llllc}
\hline & Total $(\boldsymbol{n}=\mathbf{7 2 1})$ & Severe $(\boldsymbol{n}=\mathbf{4 9 3})$ & Critical $(\boldsymbol{n}=\mathbf{2 2 8})$ & $\boldsymbol{p}$ value* \\
\hline Invasive ventilation & $103(14.3 \%)$ & $0(0 \%)$ & $103(45.2 \%)$ & $<0.0001$ \\
ECMO & $4(0.6 \%)$ & $0(0 \%)$ & $4(1.8 \%)$ & 0.016 \\
CRRT & $40(5.5 \%)$ & $1(0.2 \%)$ & $39(17.1 \%)$ & $<0.0001$ \\
\hline
\end{tabular}

$A R D S$ acute respiratory distress syndrome, $A K I$ acute kidney injury, DIC disseminated intravascular coagulation, ECMO extracorporeal membrane oxygenation, $C R R T$ continuous renal replacement therapy

a There are total 459 out of 721 cases of severe and critical cases with at least one comorbidity, including diabetes, hypertension, cancer, and cardiac disease; the percentage represents the proportion of patients with the comorbidity in their subgroup or total severe and critical cases

${ }^{*} p<0.05$ is statistically significant

may be related to prolonged bed rest during hospitalization.

In this cohort, most of the treatment did not seem to improve the outcome. Patients who had invasive ventilation even had higher mortality. Four patients received ECMO treatment, and only one of them survived. Meanwhile, only two out of 39 patients who had undergone CRRT survived. Among the drug therapies, antibiotics, antivirals, corticoids, albumin, and gamma-globulin all failed to improve the prognosis. However, anticoagulation treatment was related to a decreased death rate of critical cases (71.3\% to $88.7 \%)$. Univariate and multivariate logistic regression analyses showed that the occurrence of thrombotic disease was significantly associated with poor prognosis, and anticoagulant therapy can significantly improve prognosis (Table 2).

On the basis of the length of hospitalization, we further divided the critical disease group into three subgroups: early (died within 1 week after admission), medium (died from 1 to 2 weeks after admission), and late (died over 2 weeks after admission). Compared to early death cases, a prolonged course of disease was associated with a higher risk of hypoproteinemia, liver injury, thrombocytopenia, anemia, DIC, coagulation disorders, AKI, and infection. Higher percentages of medium and late death cases had invasive ventilation during hospitalization. Additionally, fewer of the early death cases received extra medical care, such as CRRT, antibiotics, corticosteroids, and anticoagulation (Supplementary Table 2).

As shown in Fig. 1, the early death group showed higher CK and LDH at admission and a rapid increase in CK shortly after admission, which means that high levels of $\mathrm{CK}$ and $\mathrm{LDH}$, two indicators of heart damage, may signify a high mortality risk at admission. Compared to non-survivors of critical cases, the survivors group showed a higher platelet count at admission, while most of the other indexes were not worse than the other two groups at admission (Supplementary Table 3, Supplementary Figs. 1 and 2). Notably, in late death cases, hemoglobin and platelet count decreased rapidly at the end of the observation, with a rapid deterioration in renal function (BUN and Scr), cardiac function (LDH and CK), coagulation function (PT and APTT), and inflammatory indexes (CRP, neutrophil count, and PCT) (Fig. 1, Table 3, Supplementary Figs. 1 and 2).

For the survivors, most of the indexes $(\mathrm{CK}$, LDH, lymphocyte count, ALB, CRP, neutrophil counts, etc.) began to improve after 7 days of treatment (Fig. 1, Supplementary Figs. 1 and 2). As shown in Table 3, a small difference could be found between survivors and non-survivors (medium and late groups) at admission. However, after 7 days of treatment, compared to non-survivors, the survivors had higher lymphocyte and platelet counts and lower neutrophil counts and WBC counts. Lower D-dimer, higher ALB levels, and a shorter PT 


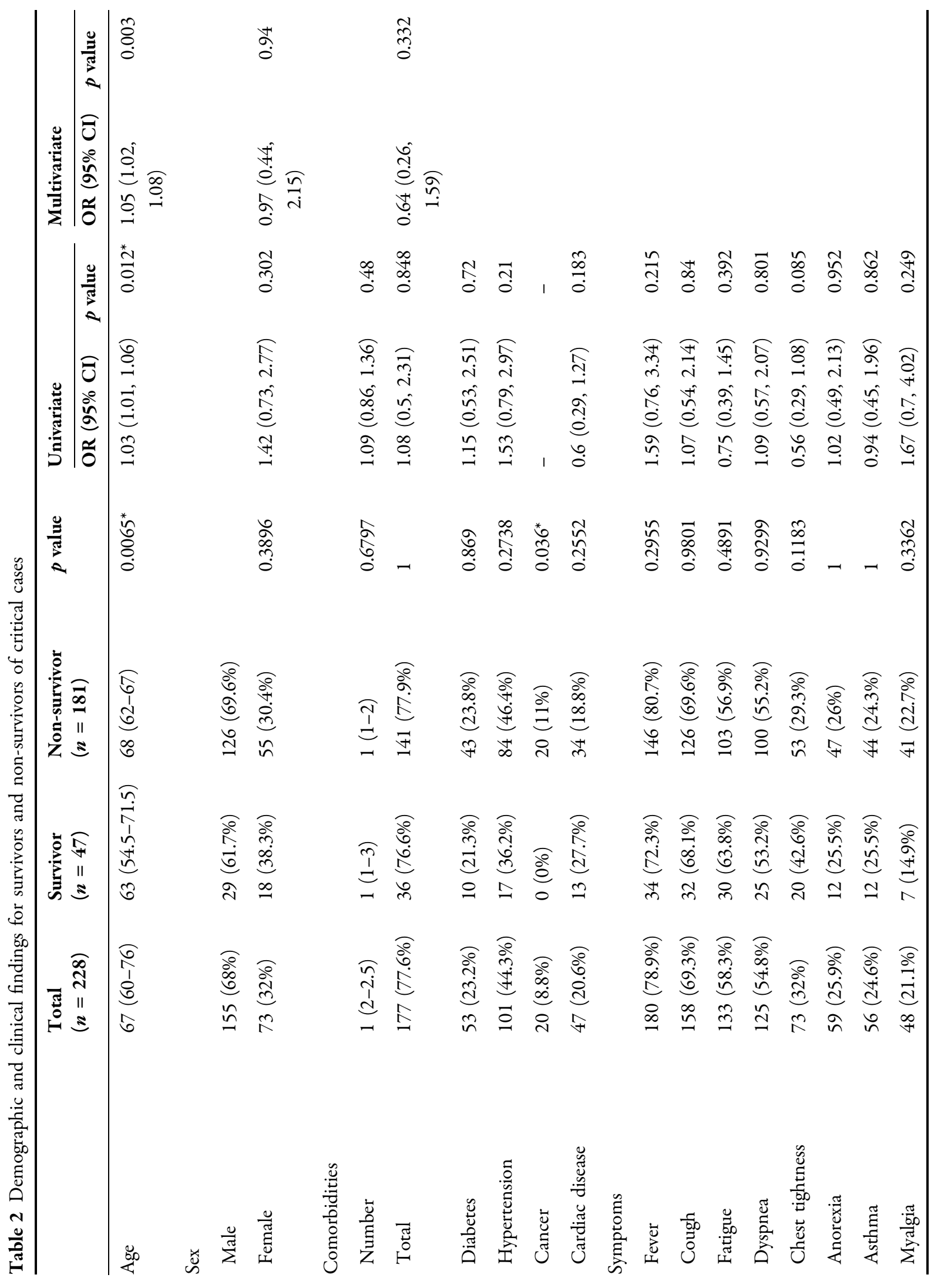




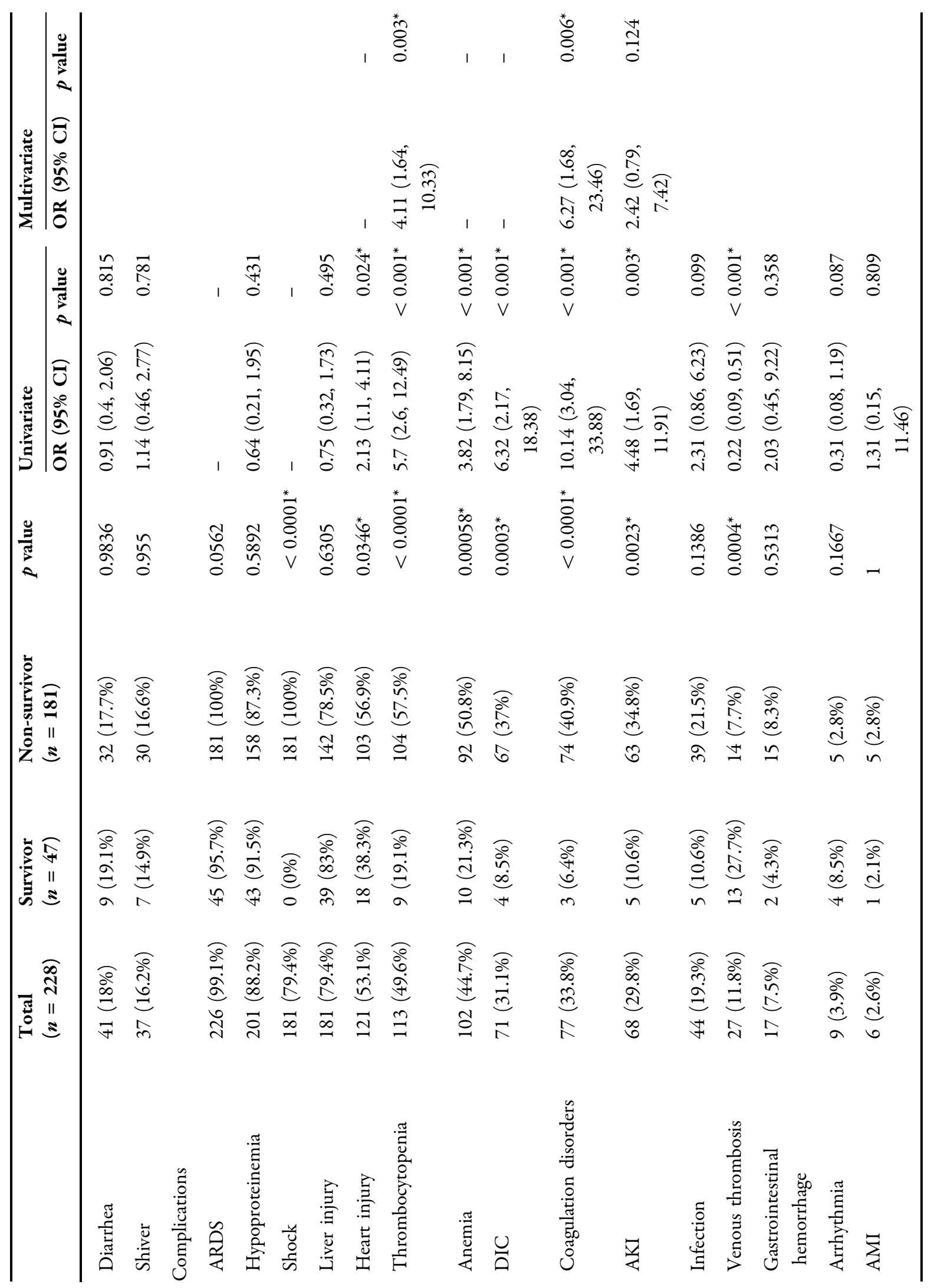




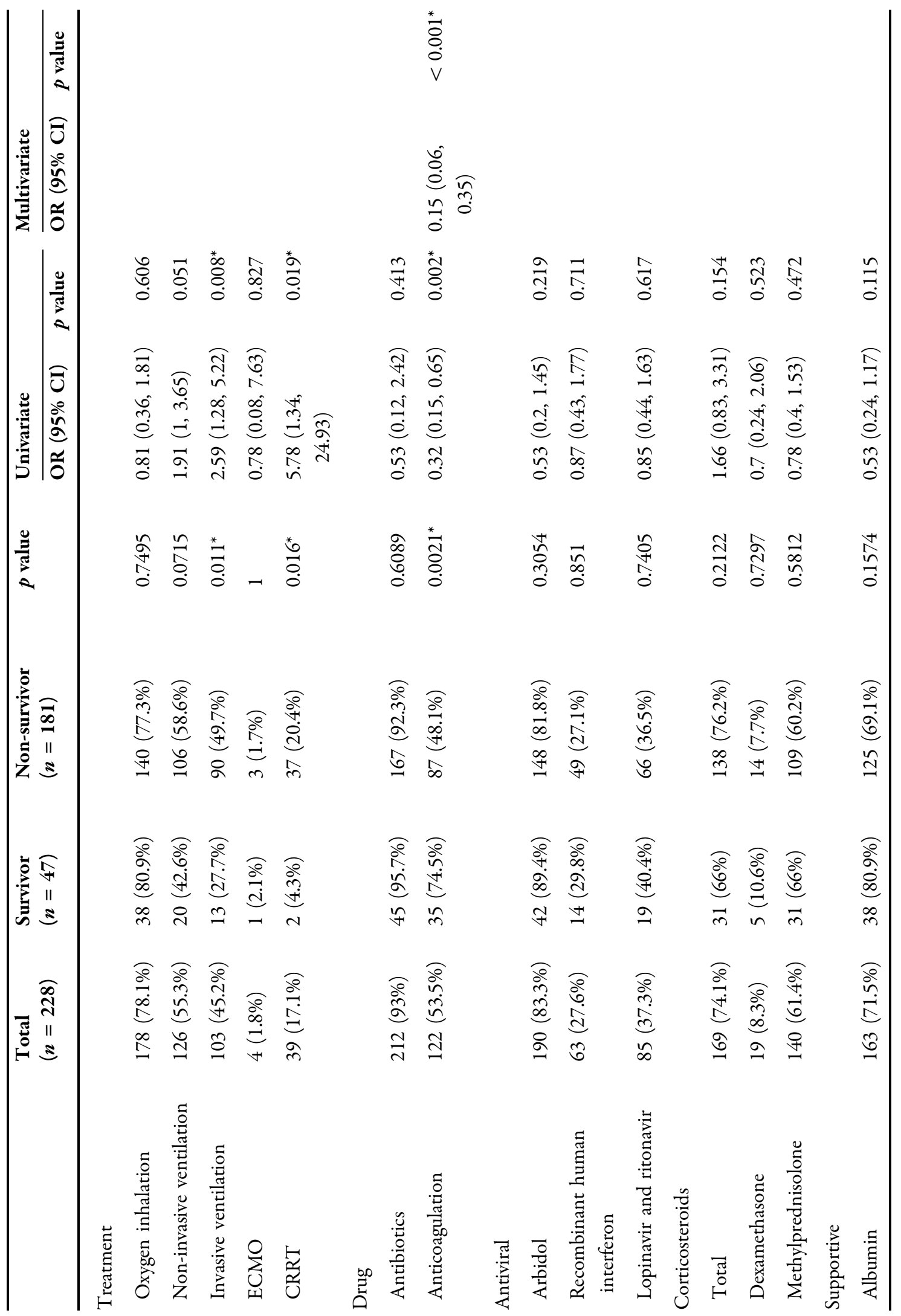




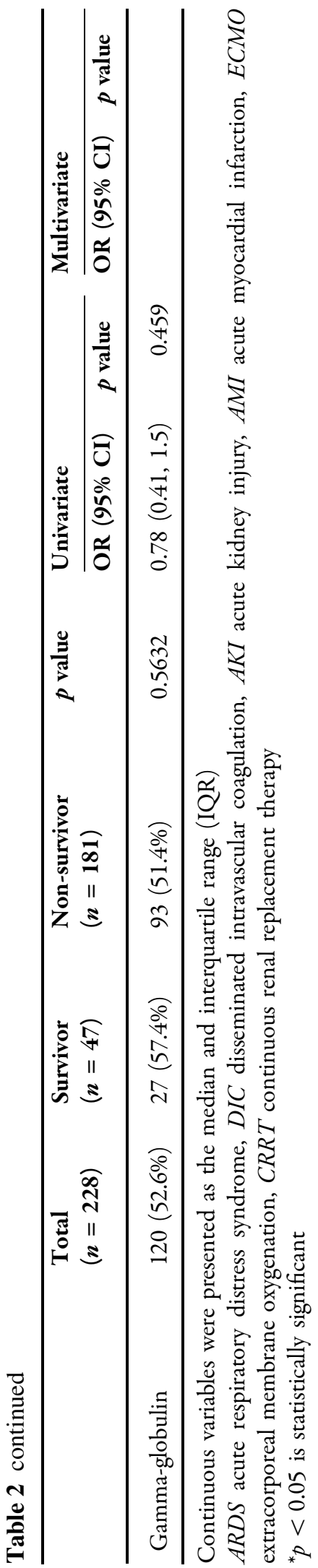

were also observed. The non-survivors also had increased BUN, cystatin C, LDH, CRP, and PCT.

In the univariate and multivariate logistic models, there were no factors associated with a poor prognosis at admission (Table 4). In contrast, on day 7 , various biochemical indexes could predict the outcome. For example, an increase in WBC and neutrophil counts indicated a poor prognosis, while platelet count, lymphocyte count, and ALB were protective factors. In addition, increases in D-dimer, BUN, and $\mathrm{LDH}$ were associated with higher odds ratios of death. This evidence indicates that the first week after admission to the hospital is the crucial period to improve the outcome of critical patients.

\section{DISCUSSION}

The outbreak of the COVID-19 pandemic has evolved into one of the most serious public health events in the last few decades. A strategy for preventing and treating COVID-19 is still pending. Previously, patients with mild COVID19 were reported to have favorable outcomes, and a considerable proportion of these cases could heal by themselves, while severe and critical cases show high mortality $[12,13]$. Since then, in-hospital medical care for severe patients has been a challenging focus for physicians [14]. In this retrospective study, we reported a summary of the clinical features of 753 COVID-19 cases, including 721 severe or critical cases, hospitalized at West Campus of Wuhan Union Hospital from January 22, 2019 to May 7, 2019. In general, older age and male sex were associated with critical disease in this cohort. Comorbidities and complications, including shock, ARDS, DIC, hepatic dysfunction, AKI, and myocardial injury, were much more frequent in critical cases.

Severe cases and critical cases show many differences, regardless of their clinical features or laboratory indexes. However, previous studies have primarily focused on the difference between all survivors and non-survivors or between severe and non-severe cases [4, 9], which mixed survivors of severe and critical cases together. This may cause confusion and 


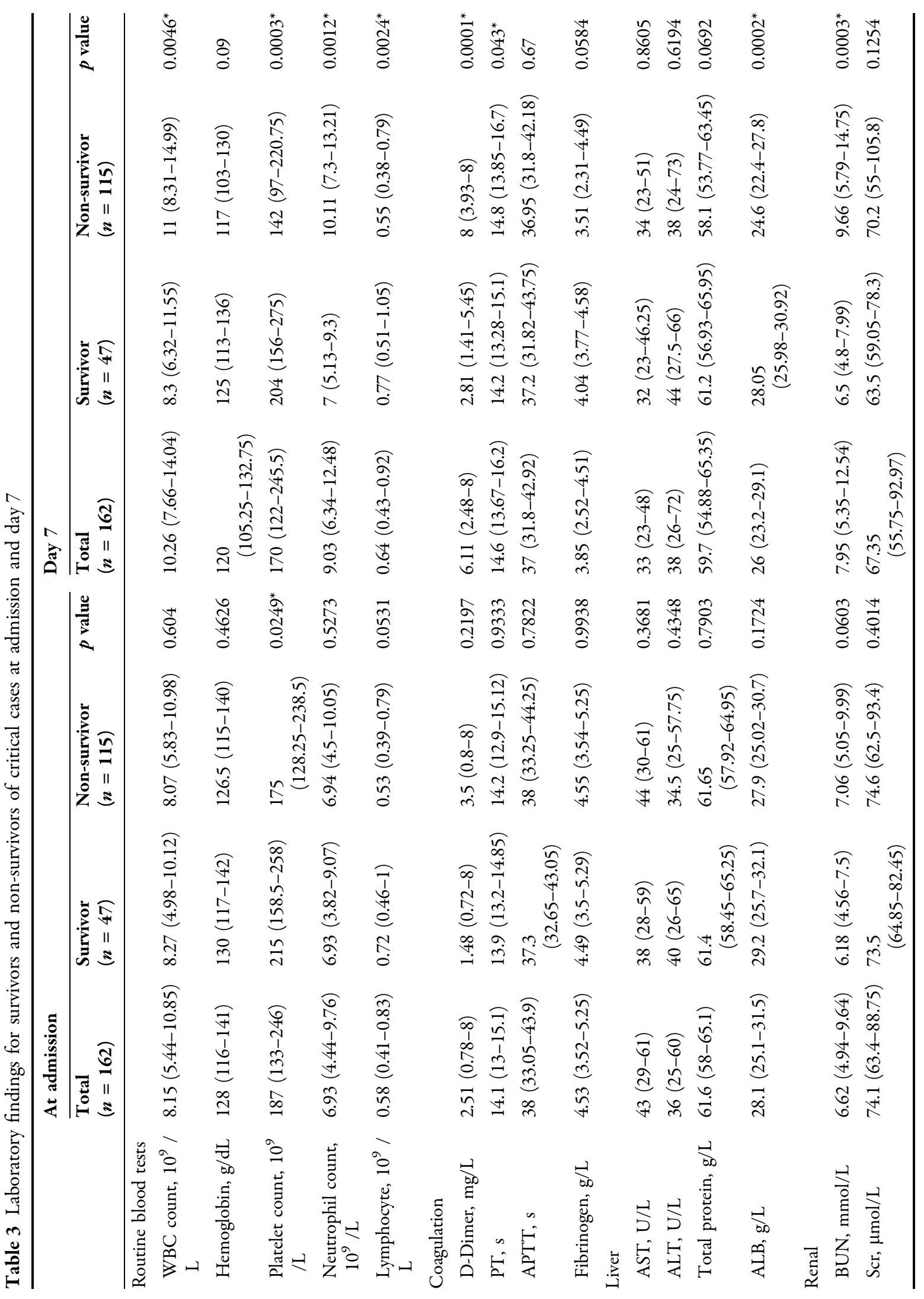




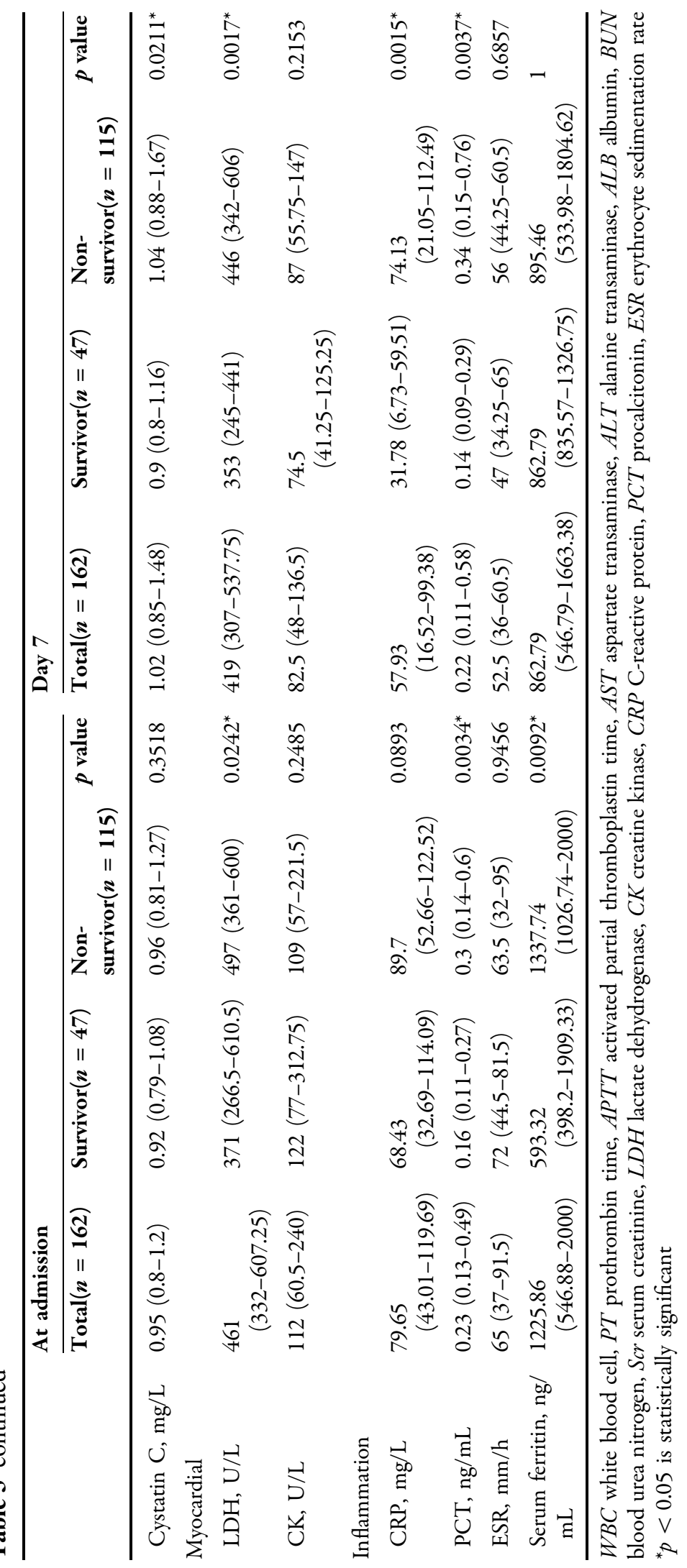



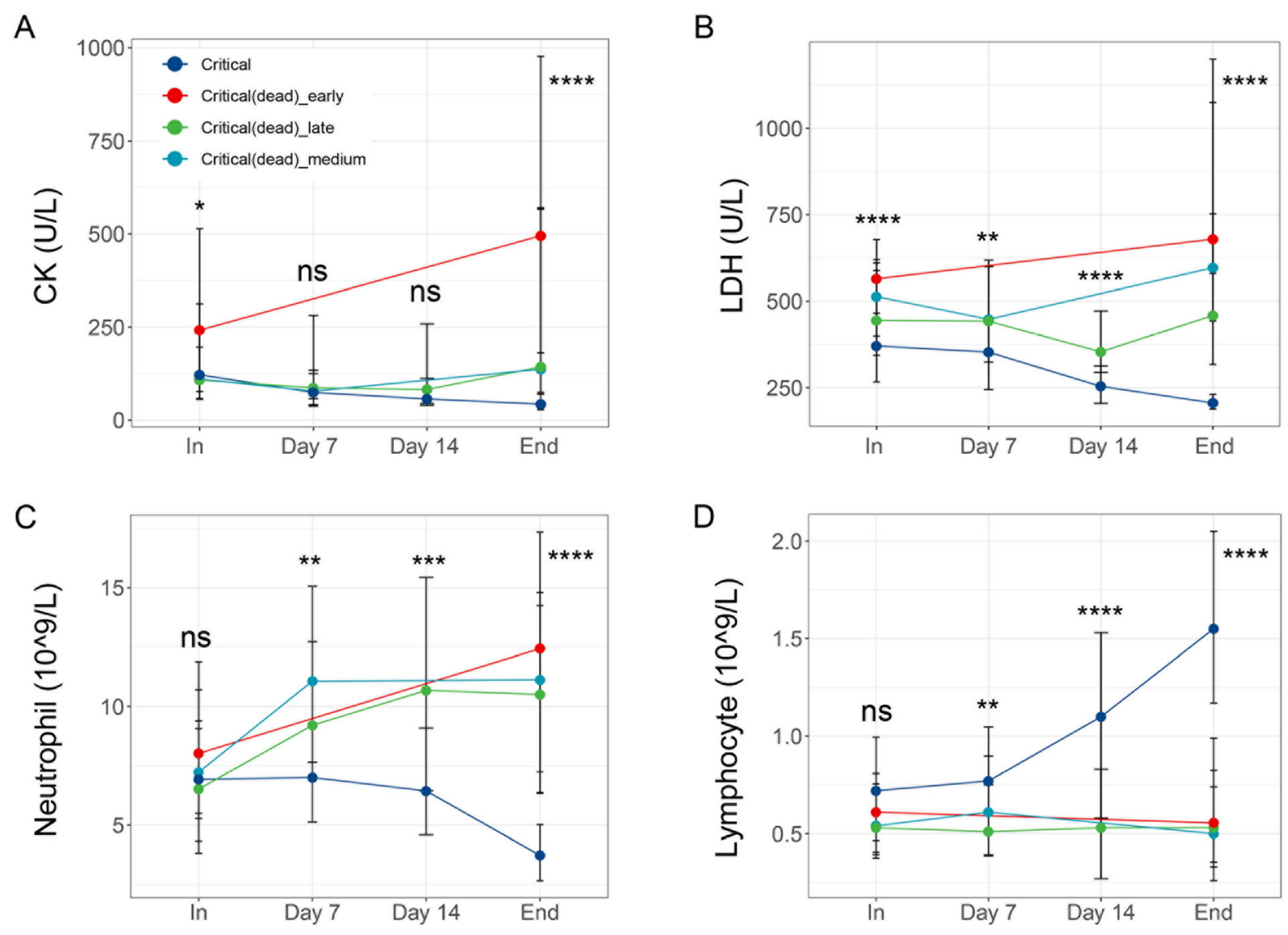

Fig. 1 Temporal changes in laboratory markers of patients with critical COVID-19 at the time of admission, 7 days after admission, 14 days after admission, and before the patients were discharge from the hospital or died.

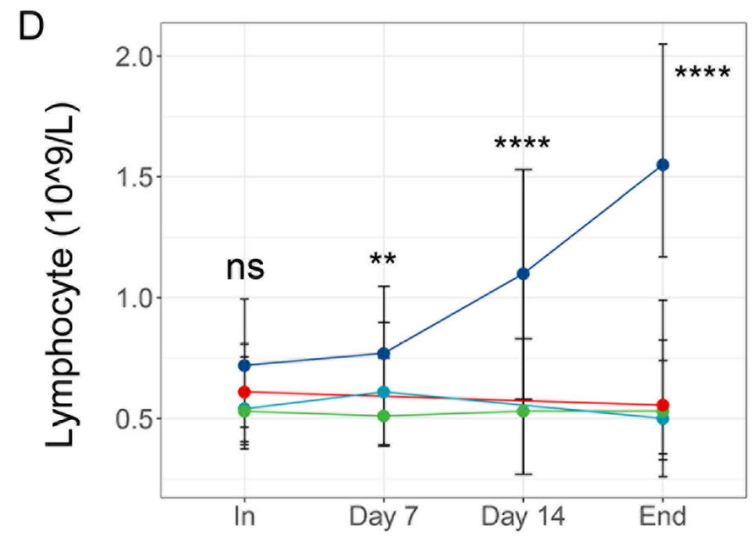

a Creatine kinase (CK); b Lactate dehydrogenase (LDH); c Neutrophil; d Lymphocyte. ${ }^{*} p<0.05$; ${ }^{* *} p<0.01 ;{ }^{* * *} p<0.001 ;{ }^{* * * *} p<0.0001 ;$ ns, $p \geq 0.05$

obscure the difference between patients with various courses of disease. Hence, we focused on survivors and non-survivors of critical cases, finding that although male patients were more likely to develop critical disease, the mortality of critical cases was not associated with sex. This was the same for comorbidities. However, age was a risk factor for death. Although all patients with underlying cancer died at the end of follow-up and this showed statistical significance, too few events were observed (only 20 participants), which may reduce the credibility of this finding. Almost all critical cases of ARDS emerged during hospitalization. Since then, immediate respiratory support is necessary for these patients. Multiorgan damage occurred in

most of the non-survivors, while the most prevalent direct cause of death was shock.

In this cohort, respiratory support, including non-invasive and invasive ventilation, did not improve the outcomes of critical cases. Invasive ventilation was even related to worse prognosis, corresponding to previous studies [15-17]. In addition, only one patient survived after ECMO treatment. Quite a few critical patients experienced AKI (29.8\%), which was correlated with poor outcomes. CRRT did not improve the outcome of these cases. All of this evidence highlights the importance of early intervention.

Most of the drug therapy failed to work in this cohort. All of the antiviral treatments seem useless against SARS-CoV-2. Although dexamethasone was reported to be able to improve the 


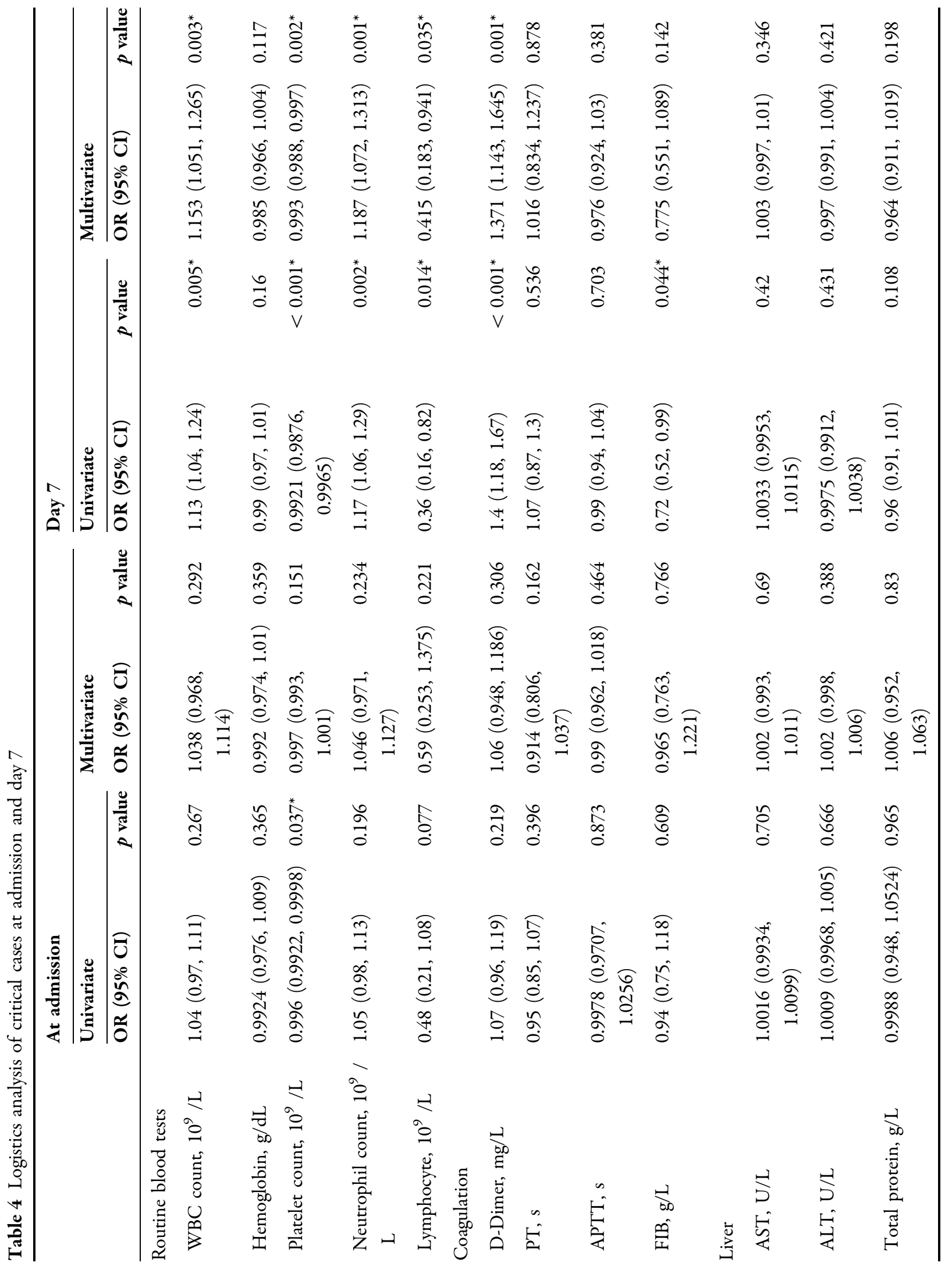




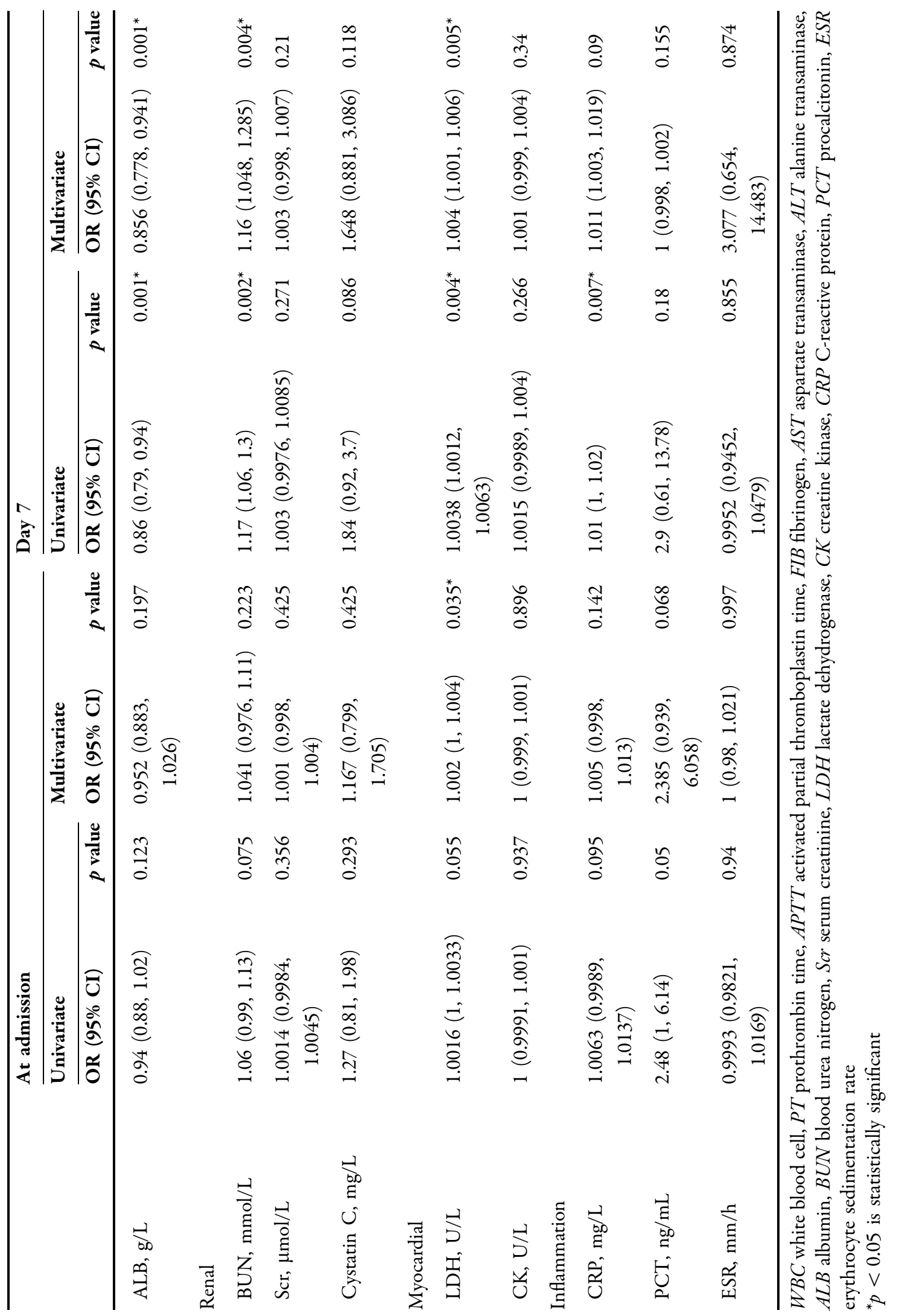


outcome of critical cases [18], it did not work in this study. This may be due to the small sample size. Strikingly, anticoagulation treatment reduced the death rate of critical patients. Compared to the early death group, the risk of coagulation disorders, venous thrombosis, and DIC significantly increased with a prolonged course of the disease. Furthermore, $27 \%$ of the survivors also experienced venous thrombosis during hospitalization. This may be caused by a continuous inflammatory response and prolonged bed rest. Dealing with the hypercoagulable state may be able to reduce death events in these patients.

Compared to medium and late non-survivors, $\mathrm{CK}$ and $\mathrm{LDH}$, especially $\mathrm{CK}$ in early death cases, were significantly higher at admission, and increased rapidly shortly after admission. This trend did not appear for the other biochemical indexes. Considering that $\mathrm{CK}$ and $\mathrm{LDH}$ are both indicators of heart injury, we suppose that heart damage or heart failure may be related to the early death risk of critical cases. However, univariate and multivariate analyses did not confirm CK as an independent predictor of early death at admission and day 7 . Univariate and multivariate analyses confirmed LDH as an independent predictor of early death and multivariate analyses confirmed it at admission. More clinical studies may be able to confirm this correlation.

Critical patients, except early death cases, did not show many differences at admission between survivors and non-survivors, which means that these cases may be curable with proper treatment strategies. Prognosis-related factors reported previously were not significant in the univariate logistic model at admission [4, 19-21]. However, this changed after 1 week of treatment, which indicates that control of the disease in the first week after admission may determine the fate of critical patients. Earlier high-grade medical care should be considered by physicians in intensive care departments.

We also noticed that hypoproteinemia, anemia, thrombocytopenia, and coagulation disorders were prevalent in patients with a longer course of disease. These complications are common in patients with terminal chronic disease, such as cancer and chronic kidney disease (CKD). They can be caused by persistent disease and could weaken the hope of recovery. Thus, additional nutritional support and blood transfusion or blood component transfusion for these patients should also be considered.

This retrospective, single-center study aimed to describe the characteristics and outcomes of 493 severe and 228 critical COVID-19 cases to analyze the risk factors and to propose a diagnosis and treatment recommendation for subsequent clinical practice. However, the critical and severe patients were treated with different treatments and the control for confounding was inadequate, affecting the reliability of this study to some extent. In addition, the lack of a treatment effect on prognosis may be related to the more severe conditions of the critical disease group (biased by indication), and further research is needed.

\section{CONCLUSIONS}

Our study revealed considerable differences between patients with severe and critical COVID-19. We found that LDH is an independent predictor of early death in critical cases, and anticoagulation therapy was correlated with an improved prognosis of patients with critical COVID-19. During the course of COVID19 disease in the critical disease group, the incidence of hypoproteinemia, anemia, thrombocytopenia, and coagulation disorders increased significantly, which highlighted the importance of medical care in the first week after admission. In addition, considering persistent disease effects, additional nutritional support and blood transfusions should be considered to improve the prognosis. Our study will help physicians understand the disease progression of critical patients and to develop proper treatment strategies.

\section{ACKNOWLEDGEMENT}

We thank the participants of the study.

Funding This work was supported by grant from the National Natural Science Foundation 
of China (NO. 81570657). The Rapid Service Fees were funded by the authors.

Authorship All named authors meet the International Committee of Medical Journal Editors (ICMJE) criteria for authorship for this article, take responsibility for the integrity of the work as a whole, and have given their approval for this version to be published.

Disclosures Zhaohui Chen, Junyi Hu, Lilong Liu, Youpeng Zhang, Dandan Liu, Ming Xiong, Yi Zhao, Ke Chen and Yu-Mei Wang declare that they have no conflict of interest.

Compliance with Ethics Guidelines This study was a retrospective study and has received approval from the Research Ethics Commission of Tongji Medical College, Huazhong University of Science and Technology (S100). The study was performed in accordance with the Helsinki Declaration of 1964, and its later amendments.

Data Availability The datasets generated during and/or analyzed during the current study are available from the corresponding author on reasonable request.

Open Access. This article is licensed under a Creative Commons Attribution-NonCommercial 4.0 International License, which permits any non-commercial use, sharing, adaptation, distribution and reproduction in any medium or format, as long as you give appropriate credit to the original author(s) and the source, provide a link to the Creative Commons licence, and indicate if changes were made. The images or other third party material in this article are included in the article's Creative Commons licence, unless indicated otherwise in a credit line to the material. If material is not included in the article's Creative Commons licence and your intended use is not permitted by statutory regulation or exceeds the permitted use, you will need to obtain permission directly from the copyright holder. To view a copy of this licence, visit http://creativecommons.org/licenses/by$\mathrm{nc} / 4.0 /$.

\section{REFERENCES}

1. WHO. Rolling updates on coronavirus disease (COVID-19). https://www.who.int/emergencies/ diseases/novel-coronavirus-2019. Accessed 10 Oct 2020.

2. Chu DK, Akl EA, Duda S, Solo K, Yaacoub S, Schünemann HJ. Physical distancing, face masks, and eye protection to prevent person-to-person transmission of SARS-CoV-2 and COVID-19: a systematic review and meta-analysis. Lancet. 2020;395(10242):1973-87.

3. Yang X, Yu Y, Xu J, et al. Clinical course and outcomes of critically ill patients with SARS-CoV-2 pneumonia in Wuhan, China: a single-centered, retrospective, observational study. Lancet Respir Med. 2020;8(5):475-81.

4. Zhou F, Yu T, Du R, et al. Clinical course and risk factors for mortality of adult inpatients with COVID-19 in Wuhan, China: a retrospective cohort study. Lancet. 2020;395(10229):1054-62.

5. Tian J, Yuan X, Xiao J, et al. Clinical characteristics and risk factors associated with COVID-19 disease severity in patients with cancer in Wuhan, China: a multicentre, retrospective, cohort study. Lancet Oncol. 2020;21(7):893-903.

6. Grasselli G, Zangrillo A, Zanella A, et al. Baseline characteristics and outcomes of 1591 patients infected with SARS-CoV-2 admitted to ICUs of the Lombardy Region Italy. JAMA. 2020;323(16): 1574-81.

7. Ellinghaus D, Degenhardt F, Bujanda L, et al. Genomewide association study of severe covid-19 with respiratory failure. N Engl J Med 2020;383(16): 1522-34.

8. Argenziano MG, Bruce SL, Slater CL, et al. Characterization and clinical course of 1000 patients with coronavirus disease 2019 in New York: retrospective case series. BMJ. 2020;369:m1996.

9. Huang C, Wang Y, Li X, et al. Clinical features of patients infected with 2019 novel coronavirus in Wuhan, China. Lancet. 2020;395(10223):497-506.

10. Hu Y, Sun J, Dai Z, et al. Prevalence and severity of corona virus disease 2019 (COVID-19): a systematic review and meta-analysis. J Clin Virol. 2020;127: 104371.

11. Williamson EJ, Walker AJ, Bhaskaran K, et al. Factors associated with COVID-19-related death using OpenSAFELY. Nature. 2020. https://doi.org/10. 1038/s41586-020-2521-4. 
12. Feng $Y$, Ling $Y$, Bai T, et al. COVID-19 with different severities: a multicenter study of clinical features. Am J Respir Crit Care Med. 2020;201(11):1380-8.

13. Gandhi RT, Lynch JB, Del Rio C. Mild or moderate Covid-19. N Engl J Med. 2020;383:1757-66.

14. Phua J, Weng L, Ling L, et al. Intensive care management of coronavirus disease 2019 (COVID-19): challenges and recommendations. Lancet Respir Med. 2020;8(5):506-17.

15. Richardson S, Hirsch JS, Narasimhan M, et al. Presenting characteristics, comorbidities, and outcomes among 5700 patients hospitalized with COVID-19 in the New York City Area. JAMA. 2020;323(20):2052-9.

16. Singer AJ, Morley EJ, Meyers K, et al. Cohort of four thousand four fundred four persons under investigation for COVID-19 in a New York hospital and predictors of ICU care and ventilation. Ann Emerg Med. 2020;76(4):394-404.
17. Chen T, Wu D, Chen H, et al. Clinical characteristics of 113 deceased patients with coronavirus disease 2019: retrospective study. BMJ. 2020;368:m1091.

18. Horby P, Lim WS, Emberson J, et al. Effect of dexamethasone in hospitalized patients with COVID19: preliminary report. medRxiv. 2020. https://doi. org/10.1101/2020.06.22.20137273.

19. Wu C, Chen X, Cai Y, et al. Risk factors associated with acute respiratory distress syndrome and death in patients with coronavirus disease 2019 pneumonia in Wuhan China. JAMA Intern Med. 2020;180(7):1-11.

20. Zhang J, Wang X, Jia X, et al. Risk factors for disease severity, unimprovement, and mortality in COVID19 patients in Wuhan, China. Clin Microbiol Infec. 2020;26(6):767-72.

21. Chen R, Liang W, Jiang M, et al. Risk factors of fatal outcome in hospitalized subjects with coronavirus disease 2019 from a nationwide analysis in China. Chest. 2020;158(1):97-105. 